\title{
A study of invisible neutrino decay at DUNE and its effects on $\theta_{23}$ measurement
}

\author{
Sandhya Choubey, ${ }^{a, b}$ Srubabati Goswami ${ }^{c}$ and Dipyaman Pramanik ${ }^{a}$ \\ ${ }^{a}$ Harish-Chandra Research Institute, HBNI, \\ Chhatnag Road, Jhunsi, Allahabad 211 019, India \\ ${ }^{b}$ Department of Physics, School of Engineering Sciences, \\ KTH Royal Institute of Technology, AlbaNova University Center, \\ 10691 Stockholm, Sweden \\ ${ }^{c}$ Physical Research Laboratory, Navrangpura, \\ Ahmedabad 380 009, India \\ E-mail: sandhya@hri.res.in, sruba@prl.res.in, \\ dipyamanpramanik@hri.res.in
}

ABSTRaCt: We study the consequences of invisible decay of neutrinos in the context of the DUNE experiment. We assume that the third mass eigenstate is unstable and decays to a light sterile neutrino and a scalar or a pseudo-scalar. We consider DUNE running in 5 years neutrino and 5 years antineutrino mode and a detector volume of $40 \mathrm{kt}$. We obtain the expected sensitivity on the rest-frame life-time $\tau_{3}$ normalized to the mass $m_{3}$ as $\tau_{3} / m_{3}>4.50 \times 10^{-11} \mathrm{~s} / \mathrm{eV}$ at $90 \%$ C.L. for a normal hierarchical mass spectrum. We also find that DUNE can discover neutrino decay for $\tau_{3} / m_{3}>4.27 \times 10^{-11} \mathrm{~s} / \mathrm{eV}$ at $90 \%$ C.L. In addition, for an unstable $\nu_{3}$ with an illustrative value of $\tau_{3} / m_{3}=1.2 \times 10^{-11} \mathrm{~s} / \mathrm{eV}$, the no decay case could get disfavoured at the $3 \sigma$ C.L. At $90 \%$ C.L. the expected precision range for this true value is obtained as $1.71 \times 10^{-11}>\tau_{3} / m_{3}>9.29 \times 10^{-12}$ in units of $\mathrm{s} / \mathrm{eV}$. We also study the correlation between a non-zero $\tau_{3} / m_{3}$ and standard oscillation parameters and find an interesting correlation in the appearance and disappearance channels with the mixing angle $\theta_{23}$. This alters the octant sensitivity of DUNE, favorably (unfavorably) for true $\theta_{23}$ in the lower (higher) octant. The effect of a decaying neutrino does not alter the hierarchy or CP violation discovery sensitivity of DUNE in a discernible way.

Keywords: Lifetime, Neutrino Detectors and Telescopes (experiments), Oscillation

ARXIV EPRINT: 1705.05820 


\section{Contents}

1 Introduction 1

2 Neutrino propagation in presence of decay 4

$3 \quad$ Experiment and simulation details $\quad 4$

4 Results 5

4.1 Constraining the $\nu_{3}$ lifetime 5

\begin{tabular}{ll}
4.2 Constraining $\theta_{23}$ and its octant 6 \\
\hline
\end{tabular}

$\begin{array}{lll}4.3 & \text { CP-violation and mass hierarchy sensitivity } & 15\end{array}$

5 Summary \& conclusion $\quad 16$

\section{Introduction}

Neutrino oscillation experiments have established that neutrinos are massive and there is mixing among all the three flavors. The neutrino oscillation parameters - namely the two mass squared differences $\Delta m_{21}^{2}=m_{2}^{2}-m_{1}^{2}$ and $\left|\Delta m_{31}^{2}\right|=\left|m_{3}^{2}-m_{1}^{2}\right|, m_{1}, m_{2}, m_{3}$ being the mass states and the three mixing angles $\theta_{12}, \theta_{13}$ and $\theta_{23}$ have been determined with considerable precision by global oscillation analysis of world neutrino data $[1,2]$. The unknown oscillation parameters are: (i) the neutrino mass hierarchy i.e whether $m_{3}>m_{2}>m_{1}$ leading to normal hierarchy $(\mathrm{NH})$ or if $m_{3}<m_{2} \approx m_{1}$ resulting in inverted hierarchy (IH); (ii) the octant of $\theta_{23}$ - if $\theta_{23}<45^{\circ}$ it is said to be in the lower octant (LO) and if $\theta_{23}>45^{\circ}$ it is in the higher octant (HO); (iii) the CP phase $\delta_{\mathrm{CP}}$. There are some indications of these quantities from the recent results of the ongoing experiments T2K [3] and $\mathrm{NO} \nu \mathrm{A}[4,5]$. However no clear conclusions have been reached yet. While more statistics from these experiments will be able to give some more definite ideas regarding the three unknowns, the precision determination is expected to come from the Fermilab based broadband experiment DUNE which has a baseline of $1300 \mathrm{~km}$. With a higher baseline than $\mathrm{T} 2 \mathrm{~K}$ and $\mathrm{NO} \nu \mathrm{A}, \mathrm{DUNE}$ is more sensitive to the earth matter effect which gives it an edge to determine the mass hierarchy and octant and hence $\delta_{\mathrm{CP}}$ by resolving the wrong-hierarchy/wrong-CP or wrong-octant/wrong-CP solutions. Thus, it is expected that DUNE will be able to determine the above three outstanding parameters with considerable precision with its projected run. For a recent analysis, in view of the latest $\mathrm{NO} \nu \mathrm{A}$ results see [6]. 
This capability of DUNE, to make precision measurements also makes it an ideal experiment to test sub-dominant new physics effects. One of the widely studied subdominant effect, in the context of neutrino experiments, is the "invisible decay" of neutrinos i.e. decay to sterile states. This can arise if neutrinos are: (i) Dirac particles and there is a coupling between the neutrinos and a light scalar boson [7,8]. This allows the decay $\nu_{j} \rightarrow \bar{\nu}_{i R}+\chi$ where $\bar{\nu}_{i R}$ is a right-handed singlet and $\chi$ is an iso-singlet scalar carrying a lepton number. (ii) Majorana particles with a pseudo-scalar coupling with a Majoron [9, 10] and a sterile neutrino giving rise to the decay $\nu_{j} \rightarrow \nu_{s}+J$. The Majoron should be dominantly singlet to comply with the constraints from LEP data on the $\mathrm{Z}$ decay to invisible particles. Such models have been discussed in [11]. In both the cases all the final state particles are sterile and invisible. The other possibility of "visible decay" occurs if neutrinos decay to active neutrinos [12-14]. The decay modes are $\nu_{j} \rightarrow \overline{\nu_{i}}+J$ or $\nu_{j} \rightarrow \nu_{i}+J$. For Majorana neutrinos both $\bar{\nu}_{i}\left(\nu_{i}\right)$ can interact as a flavor state $\bar{\nu}_{\alpha}\left(\nu_{\alpha}\right)$ according to $U_{\alpha i}^{2}$ and thus the decay product can be observed in the detector. For recent studies on implications of visible decay for long baseline experiments see $[15,16]$.

Neutrino decay manifests itself through a term of the form $\exp \left(-\frac{m_{i} L}{\tau_{i} E}\right)$ which gives the fraction of neutrinos of energy $E$ that decays after travelling through a distance $L$. Here, $\tau_{i}$ denotes the rest frame life time of the state with mass $m_{i}$.

Neutrino decay as a solution to explain the depletion of the solar neutrinos has been considered very early [17]. Subsequently, many authors have studied the neutrino oscillation plus decay solution to the solar neutrino problem and put bounds on the lifetime of the unstable state $[8,18-24]$. These studies considered $\nu_{2}$ to be the unstable state and obtained bounds on $\tau_{2}$. This is plausible, since, $U_{e 3}$ being relatively small, the admixture of $\nu_{3}$ state in the solar $\nu_{e}$ can be taken to be small. Note that most of these studies have been performed prior to the discovery of non-zero $\theta_{13}$. Typical bounds obtained from solar neutrino data is $\tau_{2} / m_{2}>8.5 \times 10^{-7} \mathrm{~s} / \mathrm{eV}$ [23]. A recent study performed in ref. [25] has obtained bound on both $\tau_{2}$ and $\tau_{1}$ including low energy solar neutrino data. Bound on $\tau_{2}$ or $\tau_{1}$ can also come from supernova neutrinos. The bound obtained in ref. [26] is $\tau / m>10^{5} \mathrm{~s} / \mathrm{eV}$ from SN1987A data.

Bounds on the $\nu_{3}$ lifetime have been obtained in the context of atmospheric and longbaseline (LBL) neutrinos. A pure neutrino decay solution (without including neutrino mixing into account) to the atmospheric neutrino problem was discussed in [27] and it was shown that this solution gives a poor fit to the data. Neutrino decay with non-zero neutrino mixing angle was first considered in [28, 29] where it was assumed that the state $\nu_{\mu}$ has an unstable component. It was shown that the neutrino decay with mixing can reproduce the $L / E$ distribution of the SuperKamiokande (SK) data. Later this scenario was reanalyzed in [30] using the zenith angle dependence of the SK data rather than the $L / E$ dsitribution and it was shown that this gives a poor fit to the SK data. In [28] and [30] the $\Delta m^{2}$ dependent terms were averaged out since it was assumed that $\Delta m^{2}>0.1 \mathrm{eV}^{2}$ to comply with the constraints coming from $\mathrm{K}$ decays [28]. However, these constraints can be evaded if the unstable state decays to some state with which it does not mix. Two scenarios have been considered in the literature in this context. The first analysis was done in ref. [31], where $\Delta m^{2}$ was kept unconstrained and it explicitly appeared in the 
probabilities. It was shown that if one fits SK data with this scenario then the best-fit comes with a non-zero value of the decay parameter and $\Delta m^{2} \sim 0.003 \mathrm{eV}^{2}$, implying that oscillation combined with small decay can give a better fit to the data. Later in ref. [32] the possibility that $\Delta m^{2} \ll 10^{-4} \mathrm{eV}^{2}$ was considered. In this case also the probability does not contain $\Delta m^{2}$ explicitly and the scenario correspond to decay plus mixing. In [32] it was shown that this scenario can give a good fit to the SK data but subsequent analysis by the SK collaboration revealed that the decay plus mixing scenario of [32] gives a poorer fit to data than only oscillation [33]. A global analysis of atmospheric and long-baseline neutrino data in the framework of the oscillation plus decay scenario considered in [31] was done in [34]. Although only oscillation gave the best- fit to the SK data, a reasonable fit was also obtained with oscillation plus decay. However, with the addition of the data from the LBL experiment MINOS, the quality of the fit became poorer. They put a bound on $\tau_{3} / m_{3} \geq 2.9 \times 10^{-10} \mathrm{~s} / \mathrm{eV}$ at the $90 \%$ C.L. Subsequently, analysis of oscillation plus decay scenario with unconstrained $\Delta m^{2}$ have been performed in [35] in the context of MINOS and T2K data and the bound $\tau_{3} / m_{3}>2.8 \times 10^{-12} \mathrm{~s} / \mathrm{eV}$ at $90 \%$ C.L. was obtained. ${ }^{1}$ Most of the above analyses have been performed in the two generation limit and matter effect in presence of decay, in the context of atmospheric and LBL neutrinos have not been studied. Expected constraint on $\tau_{3} / m_{3}>7.5 \times 10^{-11} \mathrm{~s} / \mathrm{eV}$ (95\% C.L.) in the context of the medium baseline reactor neutrino experiment like JUNO has been obtained in ref. [37] using a full three generation picture in vacuum.

Decay of ultrahigh energy astrophysical neutrinos have been considered by many authors [38-41]. A recent study performed in ref. [42] states that the IceCube experiment can reach a sensitivity of $\tau / m \geq 10 \mathrm{~s} / \mathrm{eV}$ for both hierarchies for $100 \mathrm{TeV}$ neutrinos coming from a source at a distance of $1 \mathrm{Gpc}$.

In this paper we study the constraints on $\tau_{3} / m_{3}$ from simulated data of the DUNE experiment assuming invisible decay of the neutrinos. We perform a full three flavor study and include the matter effect during the propagation of the neutrinos through the Earth. We obtain constraints on the life time of the unstable state from the simulated DUNE data. For obtaining this we assume the state $\nu_{3}$ to be unstable and obtain the constraints on $\tau_{3} / m_{3}$. We also study the discovery potential and precision of measuring $\tau_{3} / m_{3}$ at DUNE assuming decay is present in nature. In addition, we explore how the decay lifetime $\tau_{3}$ is correlated with the mixing angle $\theta_{23}$ leading to approximate degeneracy between the two parameters. We will study how this affects the sensitivity of DUNE to the mixing angle $\theta_{23}$ and its octant. The sensitivity of DUNE, to determine the standard oscillation parameters like the mass hierarchy and $\delta_{\mathrm{CP}}$ could also in principle get affected if the heaviest state is unstable, and we study this here.

The plan of the paper is as follows. In the next section we discuss the propagation of neutrinos through earth matter when one of the state undergoes decay to invisible states. Section 3 contains the experimental and simulation details. The results are presented in the section 4 and finally we conclude in section 5 .

\footnotetext{
${ }^{1}$ Currently $\mathrm{NO} \nu \mathrm{A}$ and $\mathrm{T} 2 \mathrm{~K}$ are taking data. It will be interesting to see the bounds from these experiments in the context of neutrino decay [36].
} 


\section{Neutrino propagation in presence of decay}

Assuming the $\nu_{3}$ state to decay into a sterile neutrino and a singlet scalar $\left(\nu_{3} \rightarrow \bar{\nu}_{4}+J\right)$, the flavor and mass eigenstates get related as,

$$
\left(\begin{array}{l}
\nu_{\alpha} \\
\nu_{s}
\end{array}\right)=\left(\begin{array}{ll}
U & 0 \\
0 & 1
\end{array}\right)\left(\begin{array}{l}
\nu_{i} \\
\nu_{4}
\end{array}\right)
$$

Here, $\nu_{\alpha}$ with $\alpha=e, \mu, \tau$ denote the flavor states and $\nu_{i}, i=1,2,3$ denote the three mass states corresponding to the active neutrinos. $U$ is the standard $3 \times 3$ PMNS mixing matrix. We assume that $m_{3}>m_{2}>m_{1}>m_{4}$ for NH while $m_{2} \approx m_{1}>m_{3}>m_{4}$ for IH. This implies that for both $\mathrm{NH}$ and $\mathrm{IH}$ the third mass state can decay to a lighter sterile state. Note that $\Delta m_{34}^{2}$ is unconstrained from meson decay bound since $m_{4}$ is the mass of a sterile state with which the active neutrinos do not mix. The effect of decay can be incorporated in the propagation equation by introducing a term $m_{3} / \tau_{3}$ in the evolution equation: ${ }^{2}$

$$
i \frac{d}{d x}\left(\begin{array}{c}
\nu_{e} \\
\nu_{\mu} \\
\nu_{\tau}
\end{array}\right)=\left[U\left[\frac{1}{2 E}\left(\begin{array}{ccc}
0 & 0 & 0 \\
0 & \Delta m_{21}^{2} & 0 \\
0 & 0 & \Delta m_{31}^{2}
\end{array}\right)-i \frac{m_{3}}{2 E \tau_{3}}\left(\begin{array}{lll}
0 & 0 & 0 \\
0 & 0 & 0 \\
0 & 0 & 1
\end{array}\right)\right] U^{\dagger}+\left(\begin{array}{lll}
A & 0 & 0 \\
0 & 0 & 0 \\
0 & 0 & 0
\end{array}\right)\right]\left(\begin{array}{c}
\nu_{e} \\
\nu_{\mu} \\
\nu_{\tau}
\end{array}\right)
$$

where the term $A=2 \sqrt{2} G_{F} n_{e} E$ represents the matter potential due to neutrino electron scattering in matter, $G_{F}$ denotes the Fermi coupling, $E$ is the energy, and $n_{e}$ the electron density. For the antineutrinos the matter potential comes with a negative sign. The fourth sterile state being decoupled from the other states except through the interaction which induces the decay, does not affect the propagation. We solve the eq. (2.2), in matter numerically using Runge-Kutta technique assuming the PREM density profile [44] for the Earth matter. In section 4 we will show the plots of the appearance channel probability $P_{\mu e}$ and disappearance channel probability $P_{\mu \mu}$ as a function of neutrino energy as well as a function of $\theta_{23}$ for a fixed neutrino energy.

\section{Experiment and simulation details}

DUNE (Deep Underground Neutrino Experiment) is a long baseline neutrino oscillation experiment proposed to be built in U.S.A.. DUNE will consist of a neutrino beam from Fermilab to Sanford Underground Research Facility [45-48]. The source of the beam will consist of a $80-120 \mathrm{GeV}$ proton beam which will give the neutrino beam eventually. There will be a 34-40 kt liquid Argon time projection chamber (LArTPC) at the end of the beam, at $1300 \mathrm{~km}$ from the source. We use the GLoBES package $[49,50]$ to simulate the DUNE experiment. In our simulation, we have used neutrino and antineutrino flux from a $120 \mathrm{GeV}, 1.2 \mathrm{MW}$ proton beam at Fermilab and a $40 \mathrm{kt}$ far detector. All our simulations are for a runtime of $5+5$ years ( 5 years in $\nu$ and 5 years in $\bar{\nu}$ ) and we take both appearance and disappearance channels into account, unless otherwise stated. For the charged current (CC) events we have used 20\% energy resolution for the $\mu$ and $15 \%$ energy resolution for the $e$.

\footnotetext{
${ }^{2}$ This implicitly assumes that the neutrino mass matrix and the decay matrix can be simultaneously diagonalised and the mass eigenstates are the same as the decay eigenstates [26, 43].
} 
The primary background to the electron appearance and muon disappearance signal events come from the neutral current (NC) background and the intrinsic $\nu_{e}$ contamination in the flux. In addition, there is a wrong-sign component in the flux. The problem of wrong-sign events is severe for the antineutrino run, since the neutrino component in the antineutrino flux is larger than the antineutrino component in the neutrino flux. We have taken all these backgrounds into account in our analyses. In order to reduce the backgrounds, several cuts are taken. The effects of these cuts have been imposed in our simulation through efficiency factor of the signal. We have included $2 \%(10 \%)$ signal (background) normalization errors and 5\% energy calibration error as systematic uncertainties. The full detector specification can be found in ref. [46] and we have closely followed it. A final comment on our treatment of the NC backgrounds is in order. Since neutrino decay is a non-unitary phenomenon, we expect that the NC events at DUNE would be impacted depending on the decay lifetime. Hence, one would expect that the NC background too would depend on the decay lifetime. However, we have checked that for standard threegeneration oscillations, the NC background is 71 events out of a total of 347 background events. In presence of decay $\left(\tau_{3} / m_{3}=1.2 \times 10^{-11} \mathrm{~s} / \mathrm{eV}\right)$, the $\mathrm{NC}$ background changes to 65 , while the total background is 338 . This implies that the $\mathrm{NC}$ background changes by about $8.5 \%$ only. We have also checked that including decay in the $\mathrm{NC}$ background changes the $\chi^{2}$ only in the first place in decimal and is not even visible in the plots. Hence, for simplicity we keep the NC background fixed at the standard value.

Throughout this paper we use the following true values for the standard oscillation parameters: $\theta_{12}=34.8^{\circ}, \theta_{13}=8.5^{\circ}, \Delta m_{21}^{2}=7.5 \times 10^{-5} \mathrm{eV}, \Delta m_{31}^{2}=2.457 \times 10^{-3} \mathrm{eV}$ and $\delta_{\mathrm{CP}}=-90^{\circ}$. The true value of $\theta_{23}$ will be specified for every plot that we will present. We marginalize our $\chi^{2}$ over $\theta_{13}, \theta_{23}, \delta_{\mathrm{CP}}$ and $\Delta m_{31}^{2}$, which are allowed to vary within their allowed $3 \sigma$ ranges. We add a Gaussian prior on $\theta_{13}$ with the current $1 \sigma$ range on this parameter. We keep the hierarchy normal in the simulated data for all the results presented in this paper. In this paper we have used "true" to specify the parameters used for generating the simulated data and "test" to specify the parameters in the fit.

\section{Results}

We will first present results on how well DUNE will be able to constrain or discover the lifetime of the unstable $\nu_{3}$ state. We will then turn our focus on how the measurement of standard oscillation physics at DUNE might get affected if $\nu_{3}$ were to be unstable.

\subsection{Constraining the $\nu_{3}$ lifetime}

The left panel of figure 1 shows the potential of DUNE to constrain the lifetime of $\nu_{3}$ normalized to its mass $m_{3}$. In order to obtain this curve we generate the data for a nodecay case and fit this data for an unstable $\nu_{3}$. The data was generated at the values of oscillation parameters given in section 3 and $\theta_{23}=42^{\circ}$. We marginalize the $\chi^{2}$ over all standard oscillation parameters as mentioned above. We see that at the $3 \sigma$ level DUNE could constrain $\tau_{3} / m_{3}>2.38 \times 10^{-11} \mathrm{~s} / \mathrm{eV}$, whereas at $90 \%$ C.L. the corresponding expected limit would be $\tau_{3} / m_{3}>4.50 \times 10^{-11} \mathrm{~s} / \mathrm{eV}$. This can be compared with the current limit 

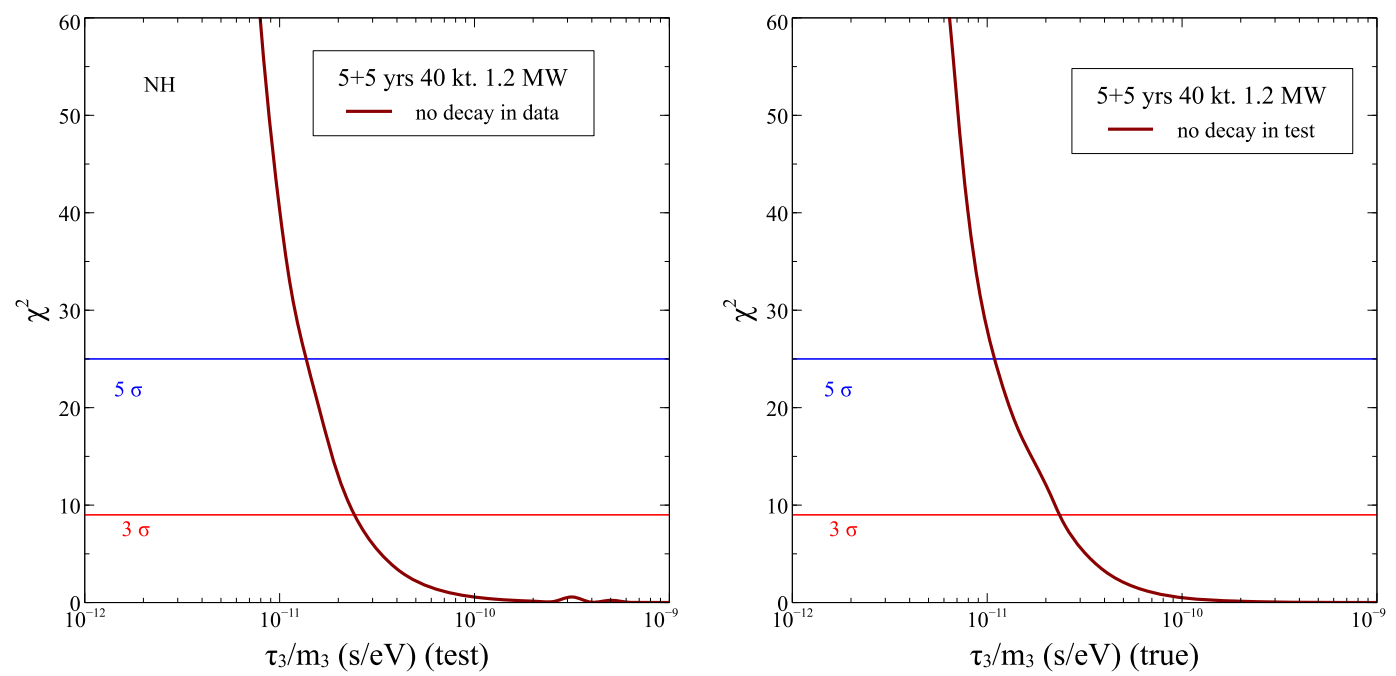

Figure 1. The left panel of this figure shows the expected sensitivity of DUNE to constraining the decay parameter $\tau_{3} / m_{3}$. The right panel of this figure presents the potential of DUNE to discover a decaying $\nu_{3}$. See text for details.

on $\tau_{3} / m_{3}>2.8 \times 10^{-12} \mathrm{~s} / \mathrm{eV}$ that we have from combined MINOS and T2K analysis [35]. Therefore, DUNE is expected to improve the bounds on $\nu_{3}$ lifetime by at least one order of magnitude. ${ }^{3}$ The right panel of figure 1 shows the discovery potential of a decaying neutrino at DUNE. To obtain this curve we generated the data taking a decaying $\nu_{3}$ into account and fitted it with a theory for stable neutrinos. True value of $\theta_{23}=42^{\circ}$ for this plot and the other oscillation parameters are taken as before. The $\chi^{2}$ so obtained is then marginalized over the standard oscillation parameters in the fit. The resultant marginalized $\chi^{2}$ is shown in the right panel of figure 1 as a function of the $\tau_{3} / m_{3}$ (true). DUNE is expected to discover a decaying neutrino at the $3 \sigma$ C.L. for $\tau_{3} / m_{3}>2.38 \times 10^{-11} \mathrm{~s} / \mathrm{eV}$ and the $90 \%$ C.L. for $\tau_{3} / m_{3}>4.27 \times 10^{-11} \mathrm{~s} / \mathrm{eV}$.

Assuming that the $\nu_{3}$ is indeed unstable with a decay width corresponding to $\tau_{3} / m_{3}=1.2 \times 10^{-11} \mathrm{~s} / \mathrm{eV}$, we show in figure 2 how well DUNE will be able to constrain the lifetime of the decaying $\nu_{3}$. It is seen from the figure that in this case not only can DUNE exclude the no decay case above $3 \sigma$, but can also measure the value of the decay parameter with good precision. The corresponding $3 \sigma$ and $90 \%$ C.L. ranges are $2.63 \times 10^{-11}>\tau_{3} / m_{3}>7.62 \times 10^{-12}$ and $1.71 \times 10^{-11}>\tau_{3} / m_{3}>9.29 \times 10^{-12}$ in units of $\mathrm{s} / \mathrm{eV}$, respectively.

\subsection{Constraining $\theta_{23}$ and its octant}

Figure 3 gives the neutrino oscillation probability for both the appearance and disappearance channels. The left panels give the probability for the appearance channel while the right panels show the probability for the disappearance channel. The top panels show the

\footnotetext{
${ }^{3}$ Note that by the time DUNE will be operative, the current experiments would have improved their statistics and hence bounds on standard and non-standard parameters. Even then after the full run of these experiments, DUNE will have more sensitivity compared to the full run of NO $\nu \mathrm{A}$ and T2K [36].
} 


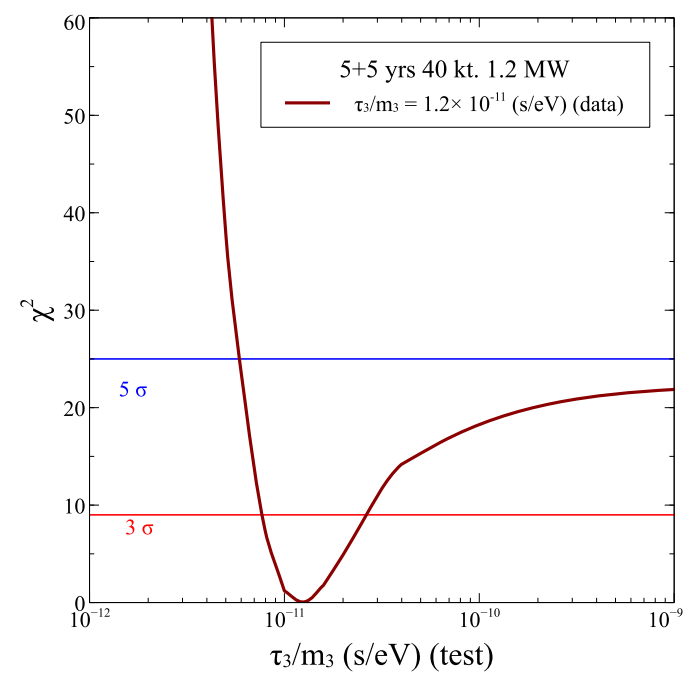

Figure 2. The $\chi^{2}$ as a function of $\tau_{3} / m_{3}$ (test), showing the precision with which $\tau_{3} / m_{3}$ could be measured at DUNE when its true value is $1.2 \times 10^{-11} \mathrm{~s} / \mathrm{eV}$.

impact of decay on the probabilities for $\tau_{3} / m_{3}=1.2 \times 10^{-11} \mathrm{~s} / \mathrm{eV}$ while the bottom panels show the effect when $\tau_{3} / m_{3}=1.0 \times 10^{-12} \mathrm{~s} / \mathrm{eV}$. The solid lines and the short-dashed lines show the probabilities for the standard stable case. The blue solid lines in all the four panels are for $\theta_{23}=42^{\circ}$ and $\theta_{13}=8.5^{\circ}$ and no decay. The change in the probabilities when decay is switched on for the same set of oscillation parameters is shown in all the panels by the red long-dashed line. The first thing to note from this figure is that $P_{\mu e}$ decreases while $P_{\mu \mu}$ increases at the oscillation maximum. The opposite trend is seen for the case when the oscillatory term goes to zero. However, net probability for both appearance as well as disappearance channels decrease in the case of decay. This is because in our model the $\nu_{3}$ decays to invisible states which do not show-up at the detector. As expected, the extent of decrease of the probability increases as we lower $\tau_{3} / m_{3}$ or in other words increase the decay rate, as can be seen by comparing the top panels with the bottom ones. For $\tau_{3} / m_{3}=1.0 \times 10^{-12} \mathrm{~s} / \mathrm{eV}$ we see a drastic change in the probability plots, thereby allowing DUNE to restrict these values of $\tau_{3} / m_{3}$, as we had seen in the previous section.

Next we turn to show the correlation between the decay lifetime $\tau_{3} / m_{3}$ and the mixing angles, in particular, the mixing angle $\theta_{23}$. In figure 3 we show that the appearance channel probability $P_{\mu e}$ for the case with decay (shown by the red long-dashed lines) can be mimicked to a large extent by the no decay scenario if we reduce the value of $\theta_{23}$. These probability curves are shown by the green short-dashed lines. For the top panels we can achieve reasonable matching between the decay and no decay scenario if the $\theta_{23}$ is reduced from $42^{\circ}$ to $41.3^{\circ}$ and $\theta_{13}$ is slightly changed from $8.5^{\circ}$ to $8.3^{\circ}$. The matching is such that the green dashed lines is hidden below the red dashed lines in the top panels. In the lower panels, since the $\nu_{3}$ lifetime is chosen to be significantly smaller, we see a more drastic effect of $\theta_{23}$. In the lower panels the with decay case for $P_{\mu e}$ at $\theta_{23}=42^{\circ}$ can be somewhat matched by the no decay case if we take a much reduced $\theta_{23}=28^{\circ}$. However, the disappearance channel is not matched between the red long-dashed and green short-dashed line for the value of $\theta_{23}$ that is needed to match the appearance channel for the decay and no decay cases. 

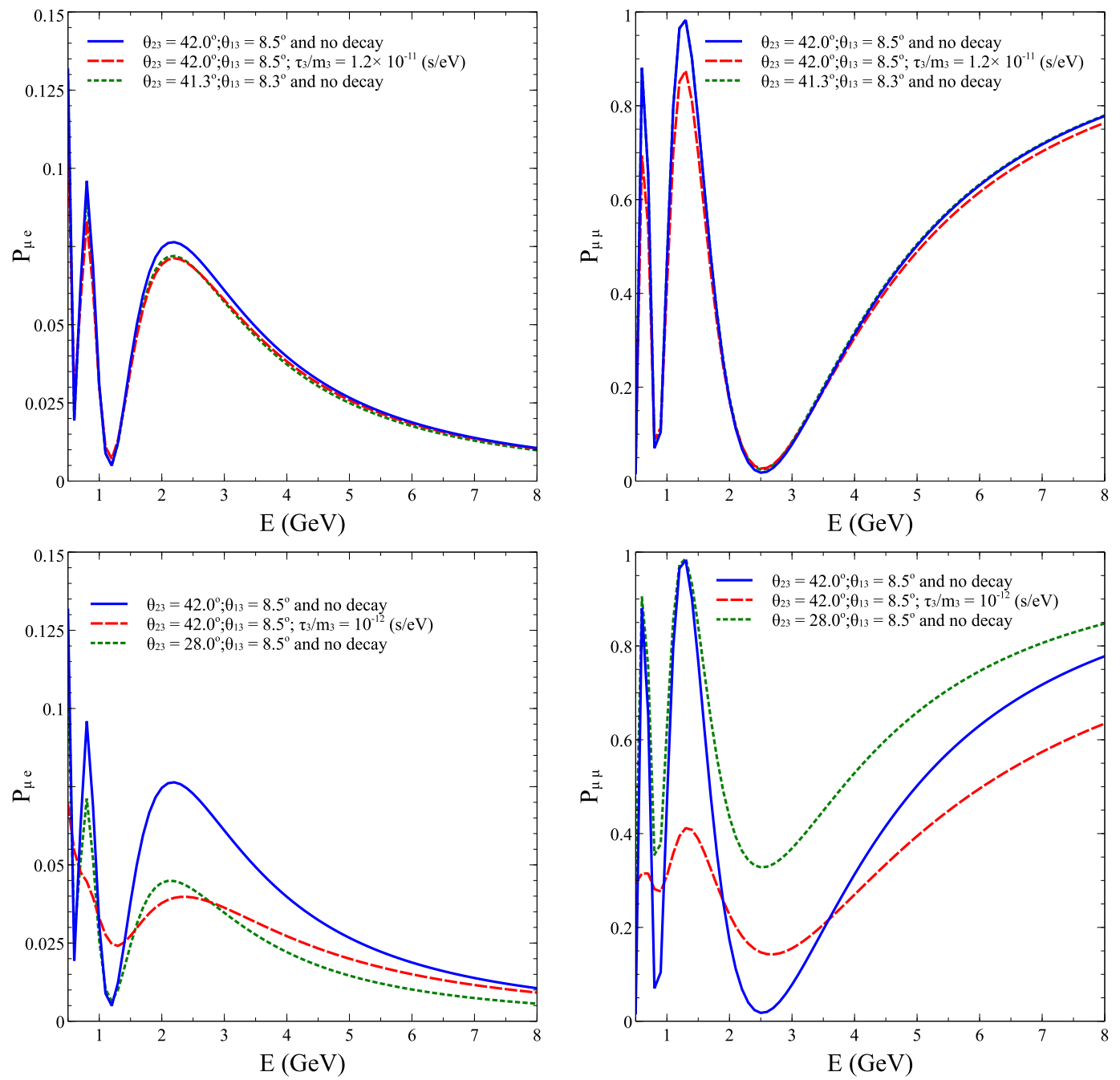

Figure 3. The appearance (left panels) and disappearance (right panels) channel neutrino probabilities as a function of neutrino energy. The different lines are described in the legends and also in the text. The top panels show the effect of $\nu_{3}$ decay for a larger value of $\tau_{3} / m_{3}$ while the bottom panels show the effect for a smaller value of $\tau_{3} / m_{3}$.

This correlation between $\tau_{3} / m_{3}$ and $\theta_{23}$ in $P_{\mu e}$ can be understood as follows. No decay corresponds to infinite $\tau_{3} / m_{3}$. As we reduce $\tau_{3} / m_{3}, \nu_{3}$ starts to decay into invisible states reducing the net $P_{\mu e}$ around the oscillation maximum. This reduction increases as we continue to lower $\tau_{3} / m_{3}$. On the other hand, it is well known that $P_{\mu e}$ increases linearly with $\sin ^{2} \theta_{23}$ at leading order. Therefore, it is possible to obtain a given value of $P_{\mu e}$ either by reducing $\tau_{3} / m_{3}$ or by reducing $\sin ^{2} \theta_{23}$. Therefore, it will be possible to compensate the decrease in $P_{\mu e}$ due to decay by increasing the value of $\sin ^{2} \theta_{23}$. Hence, if we generate the appearance data taking decay, we will be able to fit it with a theory for stable neutrinos by suitably reducing the value of $\sin ^{2} \theta_{23}$. 
The correlation between $\tau_{3} / m_{3}$ and $\theta_{23}$ for the survival channel on the other hand is complicated. For simplicity, let us understand that within the two-generation framework first, neglecting matter effects. The effect of three-generations will be discussed a little later and the effect of earth matter is not crucial for the DUNE energies in this discussion. The survival probability in the two-generation approximation is given by [35]

$$
P_{\mu \mu}^{2 G}=\left[\cos ^{2} \theta_{23}+\sin ^{2} \theta_{23} e^{-\frac{m_{3} L}{\tau_{3} E}}\right]^{2}-\sin ^{2} 2 \theta_{23} e^{-\frac{m_{3} L}{2 \tau_{3} E}} \sin ^{2}\left(\frac{\Delta m_{31}^{2} L}{4 E}\right) .
$$

The eq. (4.1) shows that decay affects both the oscillatory term as well as the constant term in $P_{\mu \mu}$, causing both to reduce. Therefore, it is not difficult to see that with decay included, the value of $\theta_{23}$ should be increased to get the same $P_{\mu \mu}$ as in the no decay case. Hence, in this case again if we generate the disappearance data taking decay, we will be able to fit it with a theory for stable neutrinos by suitably reducing the value of $\theta_{23}$. However, note that the dependence of $P_{\mu \mu}$ on $\theta_{23}$ and $\tau_{3} / m_{3}$ is different from the dependence of $P_{\mu e}$ on $\theta_{23}$ and $\tau_{3} / m_{3}$ and hence we never get the same fitted value of $\theta_{23}$ for the two channels. This is evident in figure 3 where in the lower panel the appearance probability fits between decay case and $\theta_{23}=42^{\circ}$ and no decay case and $\theta_{23}=28^{\circ}$. However, this does not fit the disappearance probability simultaneously. One can check that the above understanding of the correlation between $\theta_{23}$ and $\tau_{3} / m_{3}$ is true for the full three-generation case too. We will show below the probabilities for the full three-generation case with decay and matter effects obtained by an exact numerical computation.

In order to see the correlation between $\tau_{3} / m_{3}$ and $\theta_{23}$ at the event level, we plot in figure 4 the appearance (electron) and disappearance (muon) events for 5 years of running of DUNE in the neutrino channel. One will expect a similar behaviour for the antineutrinos as well. The respective panels and the three plots in each panel are arranged in exactly the same way as in figure 3 . We note that all the features that were visible at the probability level in figure 3 are also seen clearly at the events level in figure 4. Neutrino events are seen to reduce with the onset of neutrino decay, with the extent of reduction increasing sharply with the value of the decay rate $\left(1 / \tau_{3}\right)$. The electron event spectrum for the case with decay can be seen to be roughly mimicked with that without decay but with a lower value of the $\theta_{23}$, the required change in the value of $\theta_{23}$ increasing with the decay rate $\left(1 / \tau_{3}\right)$. On the other hand for the lower panel, the muon spectrum in absence of decay (shown by the green lines) would need a different value of $\theta_{23}$ to match the muon spectrum in presence of decay (shown by the red lines). This mismatch between the fitted value of $\theta_{23}$ between the appearance and disappearance channels can hence be expected to be instrumental in breaking the approximate degeneracy between $\tau_{3} / m_{3}$ and $\theta_{23}$.

In order to study the impact of decay on the expected $\theta_{23}$ sensitivity of DUNE, we show in figure 5 the $\chi^{2}$ as a function of $\theta_{23}$ (test). The left panel is for the case when the data is generated at $\theta_{23}=42^{\circ}$, middle panel is for data at $\theta_{23}=48^{\circ}$ and the right panel is for data at $\theta_{23}=49.3^{\circ}$. The dark red solid curves are for the standard case when both data and fit are done within the three-generation framework of stable neutrinos. The green dashed curves are for the case when the data is generated for unstable $\nu_{3}$ with $\tau_{3} / m_{3}=1.2 \times 10^{-11} \mathrm{~s} / \mathrm{eV}$ but it is fitted assuming stable neutrinos. For generating the 

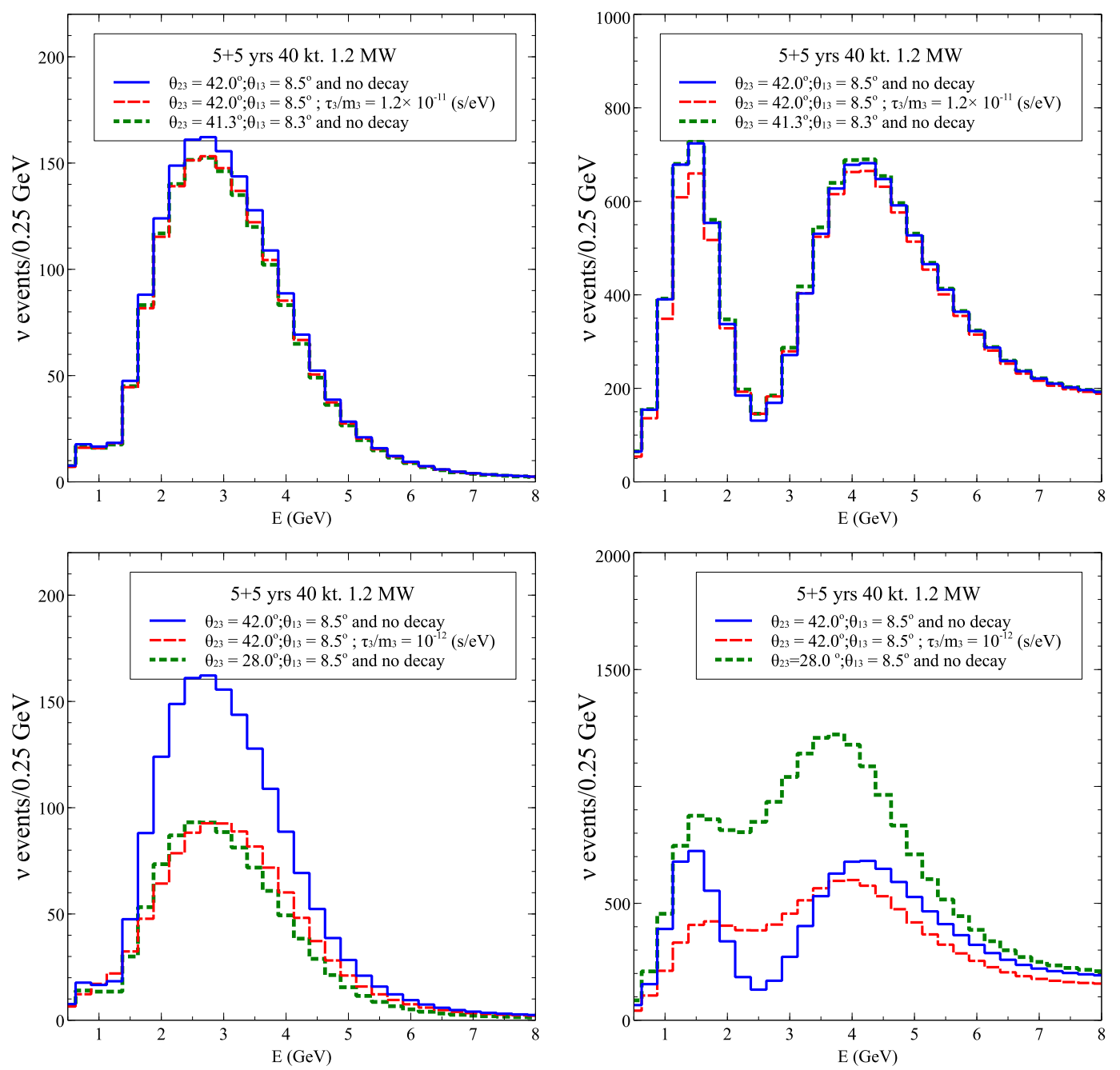

Figure 4. Same as in figure 3 but showing neutrino events at DUNE instead of probabilities. The left panels show the electron appearance events, while the right panels show the muon disappearance events. The top panels shows the impact of a larger $\tau_{3} / m_{3}$, while the bottom panels are for a smaller $\tau_{3} / m_{3}$.

data all other oscillation parameters are taken as mentioned before in section 3 . The fits are marginalised over $\theta_{13}, \delta_{\mathrm{CP}}$ and $\Delta m_{31}^{2}$ in their current $3 \sigma$ ranges. Before we proceed to look at the impact of decay on the measurement of $\theta_{23}$ at DUNE, let us expound some features of $\theta_{23}$ for standard three-generation oscillation scenario. A comparison of the red curves in the three panels of figure 5 shows that the left panel and the right panel look like near mirror images of each other, while the middle panel looks different. Note that $42^{\circ}$ is as far removed from $\theta_{23}=45^{\circ}$ as $48^{\circ}$, however the $\chi^{2}$ curves for the $\theta_{23}=42^{\circ}$ and $\theta_{23}=48^{\circ}$ cases appear different. This is due to three-generation effects coming from the non-zero $\theta_{13}$ [51]. The values of $\theta_{23}$ in $\mathrm{HO}$ and $\mathrm{LO}$ that correspond to the same effective 

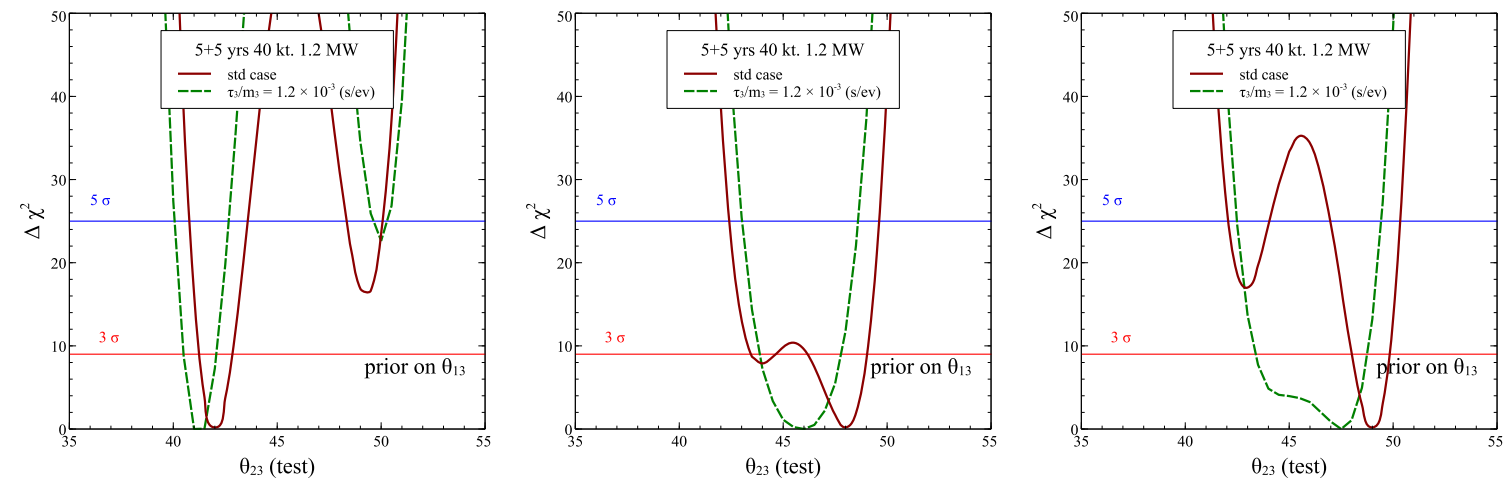

Figure 5. $\chi^{2}$ as a function of $\theta_{23}$ (test). The left, middle and right panels are for the cases when the data is generated at $\theta_{23}=42^{\circ}, \theta_{23}=48^{\circ}$ and $\theta_{23}=49.3^{\circ}$, respectively. The dark red solid curves are for the standard case when both data and fit are done within the three-generation framework of stable neutrinos. The green dashed curves are for the case when the data is generated for unstable $\nu_{3}$ with $\tau_{3} / m_{3}=1.2 \times 10^{-11} \mathrm{~s} / \mathrm{eV}$ but it is fitted assuming stable neutrinos.

mixing angle $\theta_{\mu \mu}$ and which gives the same $P_{\mu \mu}$ are given as [51]

$$
\begin{aligned}
\sin \theta_{23}^{L O} & =\frac{\sin \theta_{\mu \mu}^{L O}}{\cos \theta_{13}} ; \quad \sin \theta_{23}^{H O}=\frac{\sin \theta_{\mu \mu}^{H O}}{\cos \theta_{13}} \\
\theta_{\mu \mu}^{L O} & =90^{\circ}-\theta_{\mu \mu}^{H O},
\end{aligned}
$$

which gives $\theta_{23}=49.3^{\circ}$ as the mixing angle that gives the same $P_{\mu \mu}$ as $\theta_{23}=42^{\circ}$ instead of $\theta_{23}=48^{\circ}$, as we would expect in the two-generation case. In order to further illustrate this point, we show in figure 6 the survival probability $P_{\mu \mu}$ (blue lines) as a function of $\theta_{23}$ for the standard case (solid line) and decay case (dashed line). Also shown are the corresponding oscillation probability $P_{\mu e}$ (magenta lines) for the standard case (solid line) and decay case (dashed line). The plots have been drawn for the DUNE baseline and $E=2.5 \mathrm{GeV}$, taking all oscillation parameters as mentioned in section 3. The energy $2.5 \mathrm{GeV}$ corresponds to oscillation maximum at the DUNE baseline where the DUNE flux peaks. We note that for the standard oscillations case, $P_{\mu \mu} \simeq 0$ corresponds to a value of $\theta_{23} \simeq 46^{\circ}$ and not $45^{\circ}$ as in the two-generation case. We also note that $P_{\mu \mu}$ at $\theta_{23}=42^{\circ}$ in LO is matched by the $P_{\mu \mu}$ at $\theta_{23} \simeq 49.9^{\circ}$ in $\mathrm{HO}$, the small difference between the value of $\theta_{23}^{H O}$ derived from eq. (4.3) and the exact numerical results shown in figure 6 come from earth matter effects mainly.

The solid red curves in figure 5 showing the $\chi^{2}$ vs. $\theta_{23}$ (test) for the standard oscillation case match well with the solid blue probability curves in figure 6 . For the left panel, data is generated at $\theta_{23}=42^{\circ}$ and the absolute and fake minima come at $\theta_{23}$ (test) $=42^{\circ}$ and $49.5^{\circ}$, respectively. On the other hand for the right panel, data is generated at $\theta_{23}=49.3^{\circ}$ and the absolute and fake minima come at $\theta_{23}$ (test) $=49.3^{\circ}$ and $43.0^{\circ}$, respectively. Note that since $P_{\mu \mu}$ is nearly matched at the true and fake minima points, the disappearance data would return a $\chi^{2} \simeq 0$ at both the true as well as fake minima points giving an exact octant degeneracy. The main role of the disappearance data is only to determine the position of the minima points in $\theta_{23}$. The oscillation probability $P_{\mu e}$ on the other hand 

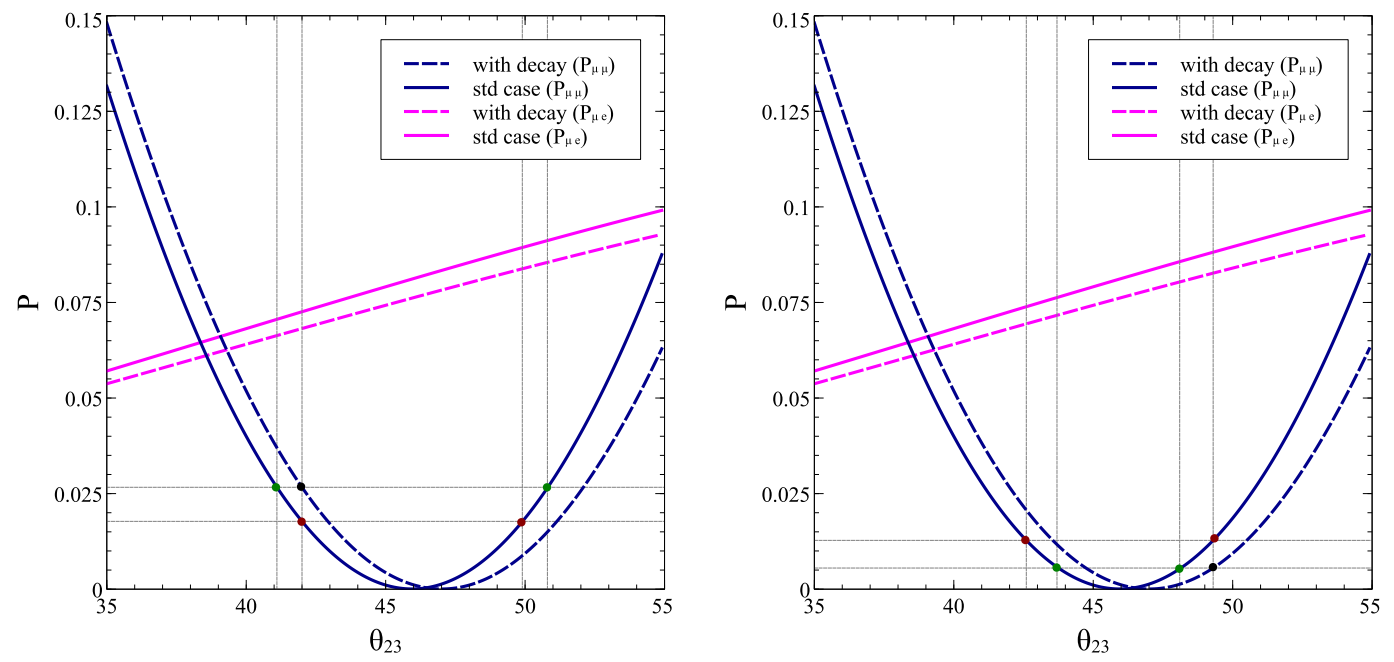

Figure 6. The probabilities $P_{\mu \mu}$ (blue lines) and $P_{\mu e}$ (magenta lines), shown as a function of $\theta_{23}$. The plots have been drawn for the DUNE baseline and $E=2.5 \mathrm{GeV}$, taking all oscillation parameters as mentioned in section 3. The left and right panels are identical apart from the horizontal and vertical lines which show the probabilities for the cases when the data is generated at $\theta_{23}=42^{\circ}$ (left panel) and $\theta_{23}=49.3^{\circ}$ (right panel). The solid curves show the probabilities for the standard case while the dashed curves are for the case for unstable $\nu_{3}$ with $\tau_{3} / m_{3}=1.2 \times 10^{-11} \mathrm{~s} / \mathrm{eV}$. The probabilities are shown for the full three-generation framework including earth matter effect. The black dot shows the point at which data is generated for the decay case, while the green dots show the points which give the same $P_{\mu \mu}$ as the black dot, but for the standard case. The red dots show the $P_{\mu \mu}$ for the data generated for the standard case at $\theta_{23}=42^{\circ}$ (left panel) and $\theta_{23}=49.3^{\circ}$ (right panel) and the corresponding fake minima when fitted by the standard case.

is very different between the true and fake minima points as can be seen from the solid magenta line in figure 6 . Hence, the appearance channel distinguishes between the two and gives a non-zero $\chi^{2}$ at the fake minima and breaks the octant degeneracy. We can see from figure 5 that for the $\theta_{23}=42^{\circ}$ case (left panel), the $\chi^{2}$ corresponding to the wrong octant minima is 16.6 while for $\theta_{23}=49.3^{\circ}$ case (right panel) it is 17.1. Hence, for standard oscillation the octant sensitivity at $\theta_{23}=42^{\circ}$ is only slightly worse than the octant sensitivity at $\theta_{23}=49.3^{\circ}$. The reason for this is that the $\chi^{2}$ for octant sensitivity is given in terms of the difference between the appearance channel event spectra for the true and fake $\theta_{23}$ points. One can see from the solid magenta lines in figure 6 that this difference is almost the same for the left and right panels for the standard oscillations case and hence the $\chi^{2}$ of the fake minima for the solid red lines in the left and right panels in figure 5 are nearly the same. The octant sensitivity for the middle panel $\left(\theta_{23}=48^{\circ}\right)$ is significantly poorer since for this case, the difference in the appearance channel probability is much smaller. This happens because this value of $\theta_{23}$ is too close to effective maximal mixing for $P_{\mu \mu}$ (cf. eqs. (4.2) and (4.3)).

Next we look at the impact of including neutrino decay in data on $\theta_{23}$ measurement at DUNE, shown by the green dashed lines in figure 5 . These lines are obtained by generating data including decay but fitting them with standard three-generation oscillations with 
stable neutrinos. We notice that compared to the red solid lines for the standard case, the position of minima as well as the $\chi^{2}$ at the fake minima have changed. For the left panel $\left(\theta_{23}=42^{\circ}\right.$ in data $)$ the minima points shift to $\theta_{23}($ test $)=41.0^{\circ}$ in $\mathrm{LO}$ and $\theta_{23}($ test $)=50.0^{\circ}$ in HO. Thus, for data with $\theta_{23}$ in LO, the minima point shifts to lower $\theta_{23}$ (test) in LO and higher $\theta_{23}$ (test) in HO. On the other hand for right panel $\left(\theta_{23}=49.3^{\circ}\right.$ in data $)$ the minima points shift to $\theta_{23}$ (test) $=44.5^{\circ}$ in $\mathrm{LO}$ and $\theta_{23}$ (test) $=47.5^{\circ}$ in $\mathrm{HO}$. Thus, for data with $\theta_{23}$ in HO, the minima point shifts to higher $\theta_{23}$ (test) in LO and lower $\theta_{23}$ (test) in HO. Note that none of the minima now correspond to the true value of $\theta_{23}$ at which the data is generated. Note also that the gap between the two minima points has increased for the case with data in LO and decreased for the case with data in HO.

The shifting of minima for both the LO and HO data points can be understood easily in terms of the left and right panels of figure 6 , respectively. This figure shows $P_{\mu \mu}$ (and $\left.P_{\mu e}\right)$ at the oscillation maximum as a function of $\theta_{23}$. The solid lines are for no decay while the dashed lines are for decay and oscillations. An important thing we can note in this figure is that with decay the $P_{\mu \mu}$ curve gets shifted towards the right. Even the effective maximal mixing point gets shifted further towards higher values of $\theta_{23}$. The left panel of figure 6 shows the data point for the disappearance channel for $\theta_{23}=42^{\circ}$ by the black point on the blue dashed line, which includes decay. This $P_{\mu \mu}$ has to be reproduced by the no decay theory in the fit. The corresponding minima points can be obtained by following the blue solid line and are shown by the green dots, at $\theta_{23}=41.1^{\circ}$ and $\theta_{23}=50.8^{\circ}$. This matches well with the minima on the green dashed line in the left panel of figure 5 . The point where data is generated for the $\mathrm{HO}$ case in figure 5 is shown by the black dot in the right panel of figure 6 . The corresponding fit points coming from $P_{\mu \mu}$ can be seen at the green dots in this panel at $\theta_{23}=43.7^{\circ}$ and $\theta_{23}=48.1^{\circ}$. Note that since the decay causes the $P_{\mu \mu}$ curve to shift towards the right, all minima points in $\theta_{23}$ are shifted towards the left. However, when one compares the minima in the true and fake octants, it turns out that the two minima get further separated for the LO case (left panel), while for the HO case (right panel) they come closer together. This is consistent with the gap between the minima increasing for the left panel and decreasing for the right panel in figure 5 . As mentioned before, the disappearance data plays the role of determining the minima in the true and fake octant, but brings no significant octant sensitivity since $P_{\mu \mu}$ can be matched at the true and fake minima, at least at the oscillation maximum. The octant sensitivity comes from the difference in the number of appearance events at the minima points at the true and fake octant. Therefore, since the minima points in the LO case gets further separated for the decay case compared to no decay, the octant sensitivity for LO increases as can be seen from the $P_{\mu e}$ curve in figure 6 . We can read from the left panel of figure 5 that the $\chi^{2}$ at the minima in the fake octant is 22.6, higher than the case for standard oscillations. On the other hand for the right panel, the minima points come closer in the case of the dashed green lines and the octant sensitivity coming from the appearance channel drops significantly to $\chi^{2}=4.1$ for the wrong octant since the difference in $P_{\mu e}$ between the minima points reduces, as can be seen from the right panel of figure 6 .

The impact of decay on the expected constraints in the two-dimensional $\theta_{23}-\theta_{13}$ plane is shown in figure 7. As in figure 5 , the left panel shows the results when the data is 

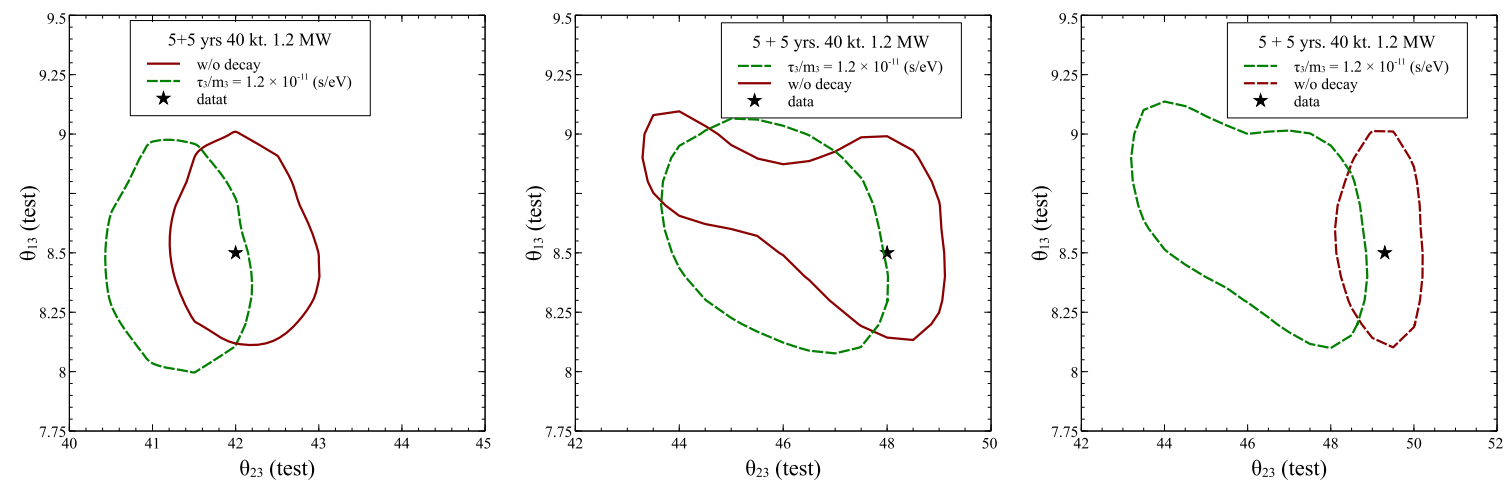

Figure 7. The plots show the expected $3 \sigma$ C.L. contours in the $\theta_{23}-\theta_{13}$ plane for the case when the data is simulated at $\theta_{23}=42^{\circ}$ (left panel), $\theta_{23}=48^{\circ}$ (middle panel), and $\theta_{23}=49.3^{\circ}$ (right panel). The value of $\theta_{13}=8.5^{\circ}$ in all panels. The black stars show the data points in the plane. The dark red solid curves show the expected $3 \sigma$ contour for the standard scenario in absence of decay in data and theory. The green dashed curves show the $3 \sigma$ contour for the case when the data corresponds to a decaying $\nu_{3}$ with $\tau_{3} / m_{3}=1.2 \times 10^{-11} \mathrm{~s} / \mathrm{eV}$, which is fitted with a theory where all neutrinos are taken as stable.

generated at $\theta_{23}=42^{\circ}$, middle panel is for $\theta_{23}=48^{\circ}$, while the right panel gives the results for data corresponding to $\theta_{23}=49.3^{\circ}$. The point where the data is generated is marked by a star in the $\theta_{23}-\theta_{13}$ plane. The expected contours correspond to $3 \sigma$ C.L. The dark red solid lines are obtained for the standard case when neutrinos are taken as stable in both the data as well as the fit. The green dashed ones are obtained when we simulate the data assuming an unstable $\nu_{3}$ with $\tau_{3} / m_{3}=1.2 \times 10^{-11} \mathrm{~s} / \mathrm{eV}$, but fit it with the standard case assuming stable neutrinos. The contours are marginalized over test values of $\delta_{\mathrm{CP}}$ and $\Delta m_{31}^{2}$ within their current $3 \sigma$ ranges. The impact of decay is visible in all panels. Though the contours change in both mixing angles, the impact on $\theta_{23}$ (test) is seen to be higher than the impact on $\theta_{13}$ (test). As we had seen in details above in figure 5 , the green contours are shifted to lower values of $\theta_{23}$ in both the left and right panels. The one-to-one correspondence between the allowed $\theta_{23}$ (test) values at $3 \sigma$ between this figure and figure 5 can be seen. The mild anti-correlation between the allowed values of $\theta_{23}$ (test) and $\theta_{13}$ (test) for the green dashed lines comes mainly from the appearance channel which depends on the product of $\sin ^{2} \theta_{23} \sin ^{2} 2 \theta_{13}$ at leading order. This anti-correlation is seen to be more pronounced for the middle and right panels because for these cases the $\theta_{23}$ sensitivity of the data falls considerably in presence of decay and the $\chi^{2}$ drops.

The figure 8 shows the octant sensitivity for $5+5$ years of $(\nu+\bar{\nu})$ running of DUNE. The dark-red solid curve shows the octant sensitivity for the standard case with stable neutrinos. The green dashed curve is for the case when $\nu_{3}$ is taken as unstable with $\tau_{3} / m_{3}=1.2 \times 10^{-11} \mathrm{~s} / \mathrm{eV}$ in the data, but in the fit we keep it to be stable. We note that the octant sensitivity of DUNE improves for the green dashed line in the lower octant, but in the higher octant it deteriorates. This is consistent with our observations in figure 5 . For detailed explanation of this, we refer the reader to the detailed discussion above. 


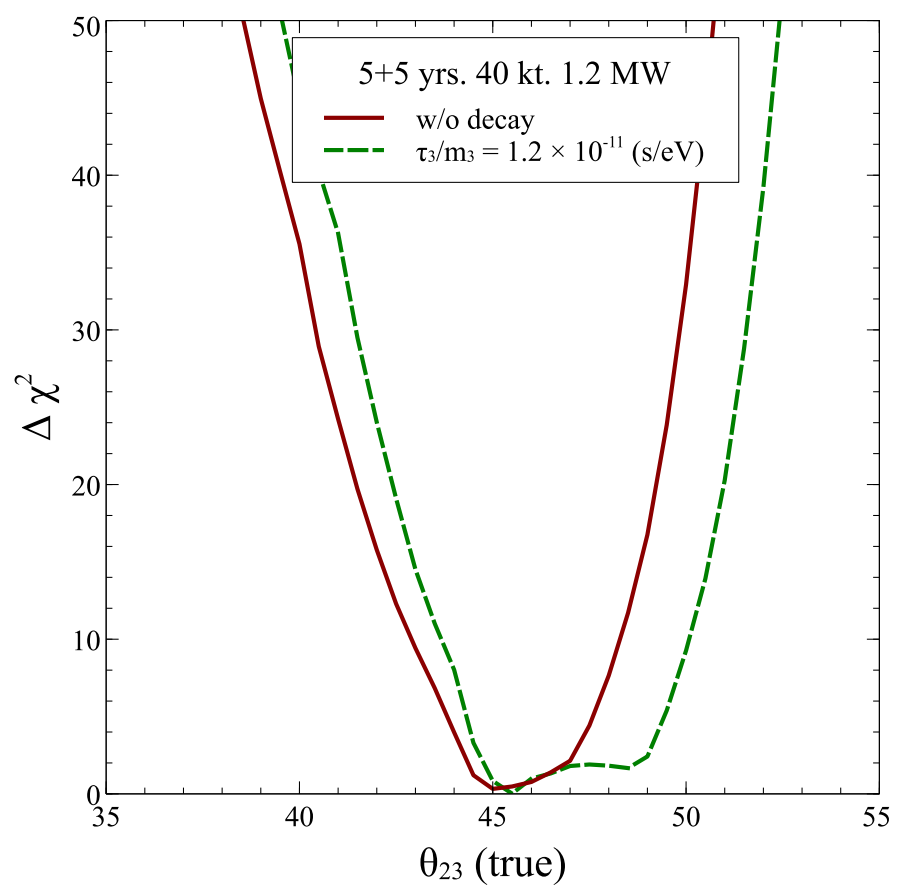

Figure 8. Expected octant sensitivity at DUNE. The dark red solid curve is for standard case of stable neutrinos. The green dashed curve is for the case when $\nu_{3}$ is taken as unstable with $\tau_{3} / m_{3}=1.2 \times 10^{-11} \mathrm{~s} / \mathrm{eV}$ in the data, but in the fit we keep it to be stable.

\subsection{CP-violation and mass hierarchy sensitivity}

In figure 9 we show the expected CP-violation sensitivity at DUNE. As before, the dark red solid curve is for standard case of stable neutrinos. The green dashed curve is for the case when $\nu_{3}$ is taken as unstable with $\tau_{3} / m_{3}=1.2 \times 10^{-11} \mathrm{~s} / \mathrm{eV}$ in the data, but in the fit we keep it to be stable. The data was generated at the values of oscillation parameters given in section 3 and $\theta_{23}=42^{\circ}$. Decay in the data is seen to bring nearly no change to the CP-violation sensitivity of DUNE, with only a marginal increase in the CP-violation sensitivity seen at $\delta_{\mathrm{CP}}($ true $) \simeq \pm 90^{\circ}$.

The impact of decay on the expected mass hierarchy sensitivity at DUNE is shown in figure 10 for both normal hierarchy (NH) true (left panel) and inverted hierarchy (IH) true (right panel). As in all figures shown so-far, the dark red solid curve is for standard case of stable neutrinos. The data was generated at the values of oscillation parameters given in section 3 and $\theta_{23}=42^{\circ}$. The green dashed curve is for the case when $\nu_{3}$ is taken as unstable with $\tau_{3} / m_{3}=1.2 \times 10^{-11} \mathrm{~s} / \mathrm{eV}$ in the data, but in the fit we keep it to be stable. For IH true, the effect of decay in data is to marginally reduce the expected mass hierarchy sensitivity for all values of $\delta_{\mathrm{CP}}$ (true). The impact for the NH true case is more complicated with the expected sensitivity increasing for some values of $\delta_{\mathrm{CP}}$ (true) and decreasing for others. However, the net change in the expected sensitivity is seen to be very small compared to the expected mass hierarchy sensitivity at DUNE. Therefore, we conclude that the expected CP-violation sensitivity and mass hierarchy sensitivity at DUNE remain largely unmodified, whether or not neutrinos decay. 


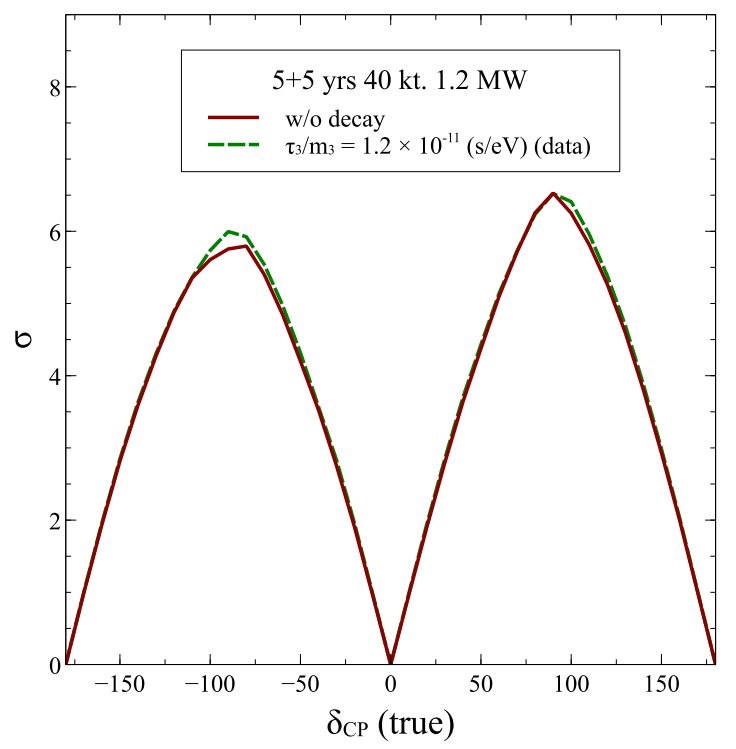

Figure 9. Expected CP-violation sensitivity at DUNE. The dark red solid curve is for standard case of stable neutrinos. The green dashed curve is for the case when $\nu_{3}$ is taken as unstable with $\tau_{3} / m_{3}=1.2 \times 10^{-11} \mathrm{~s} / \mathrm{eV}$ in the data, but in the fit we keep it to be stable.
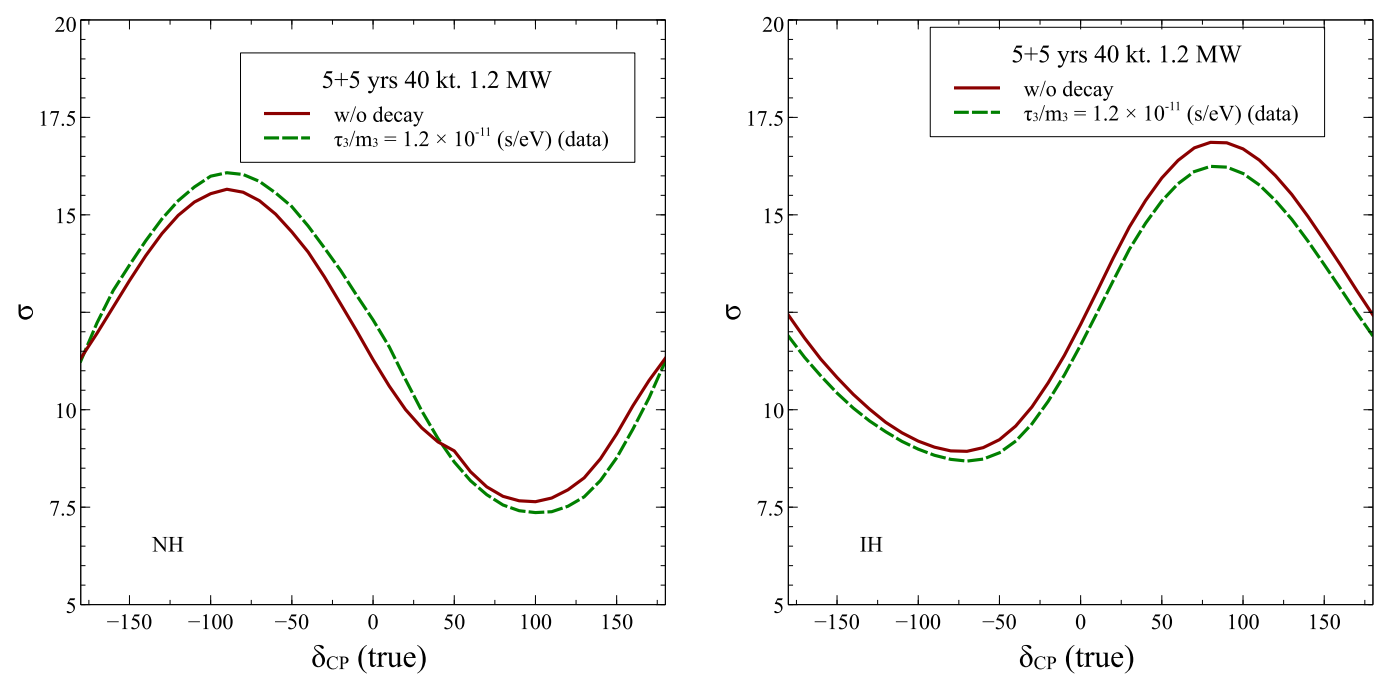

Figure 10. Expected mass hierarchy sensitivity at DUNE. The dark red solid curve is for standard case of stable neutrinos. The green dashed curve is for the case when $\nu_{3}$ is taken as unstable with $\tau_{3} / m_{3}=1.2 \times 10^{-11} \mathrm{~s} / \mathrm{eV}$ in the data, but in the fit we keep it to be stable. The left panel is for $\mathrm{NH}$ true while the right panel is for IH true.

\section{Summary \& conclusion}

We studied the impact of invisible neutrino decay for the DUNE experiment. We assumed that the third mass eigenstate is unstable and decays to a very light sterile neutrino. The mass of this state $m_{4}$ is assumed to be smaller than the mass of the third mass eigenstate $m_{3}$ irrespective of the hierarchy. We did a full three-generation study incorporating matter 
effects in our numerical simulations. First, we studied the sensitivity of DUNE to constrain the parameter $\tau_{3} / m_{3}$ and obtained the expected sensitivity $\tau_{3} / m_{3}>4.50 \times 10^{-11} \mathrm{~s} / \mathrm{eV}$ at $90 \%$ C.L. for NH, $5+5$ year of DUNE data and a $40 \mathrm{kt}$ detector volume. This is one order of magnitude improvement over the bound obtained in [35] from combined MINOS and $\mathrm{T} 2 \mathrm{~K}$ data. Of course the bound from $\mathrm{T} 2 \mathrm{~K}$ and $\mathrm{NO} \nu \mathrm{A}$ is expected to improve in the future, but here we have concentrated only on the prospective bounds from DUNE. Note that bound on decay from DUNE is expected to be better than that expected from the full run of current experiments. We also studied the potential of DUNE to discover neutrino decay, should it exists in nature and found that DUNE can discover a decaying neutrino scenario for $\tau_{3} / m_{3}>4.27 \times 10^{-11} \mathrm{~s} / \mathrm{eV}$ at $90 \%$ C.L. with its projected run. In addition, we explored how precisely DUNE can constrain the decay parameter and showed that for an unstable $\nu_{3}$ with $\tau_{3} / m_{3}=1.2 \times 10^{-11} \mathrm{~s} / \mathrm{eV}$, the no decay case gets excluded at $3 \sigma$. At $90 \%$ C.L. the allowed range corresponding to this true value is given as $1.71 \times 10^{-11}>\tau_{3} / m_{3}>9.29 \times 10^{-12}$ in units of $\mathrm{s} / \mathrm{eV}$.

We showed that an interesting correlation exists between the decay lifetime and the parameter $\theta_{23}$ both in the appearance probability $P_{\mu e}$ as well as the disappearance probability. For values of $\tau_{3} / m_{3}$ for which fast invisible decay relevant for the baseline under consideration occurs, the probability $P_{\mu e}$ decreases. This decrease can be compensated by a higher value of $\sin ^{2} \theta_{23}$. Alternatively, if we assume decay to be present in the data then it can be mimicked by a no decay scenario for a lower value of $\theta_{23}$ leading to an erroneous determination of the latter. Since it is well known that determination of $\theta_{23}$ is correlated with the value of $\theta_{13}$, we presented contours in the $\theta_{23}-\theta_{13}$ plane assuming decay in data and fitting it with a model with no decay. We found that the contours show a trend to move towards lower $\theta_{23}$ value. The allowed range of $\theta_{13}$ also spreads as compared to the only oscillation case, but the effect is more drastic for $\theta_{23}$.

We performed a detailed study of the correlation between decay and $\theta_{23}$ for the disappearance channel $P_{\mu \mu}$ and studied how decay affects the $\theta_{23}$ octant sensitivity in DUNE. Since the position of the minima in both the true and fake octant is determined by the disappearance data while the $\chi^{2}$ at the fake minima is determined by the appearance data, the effect of decay appears through both channels to affect the octant sensitivity at DUNE and we discussed this in detail. We showed how and why the octant sensitivity of DUNE improved for the lower octant and reduced for the higher octant. We also studied the impact of a decaying neutrino on the determination of hierarchy and $\delta_{\mathrm{CP}}$ at DUNE. The invisible decay scenario considered in this work affects the hierarchy and CP sensitivity of DUNE nominally. In conclusion, the DUNE experiment provides an interesting testing ground for the invisible neutrino decay hypothesis for $\tau_{3} / m_{3} \sim 10^{-11} \mathrm{~s} / \mathrm{eV}$.

Note added. While we were finalizing this work, ref. [15] came, which also addresses exploration of neutrino decay at DUNE. Their emphasis is more on visible decay though they also provide a comparison with the invisible decay case. We consider invisible decays to light sterile neutrinos and have explored different parameter spaces. Hence the two works supplement each other. We also discussed the impact of decay on the determination of $\theta_{23}$ and its octant. In addition we also studied the effect of decay on mass hierarchy and $\delta_{\mathrm{CP}}$ discovery at DUNE. 


\section{Acknowledgments}

We acknowledge the HRI cluster computing facility (http://www.hri.res.in/cluster/). SG would like to thank Lakshmi. S. Mohan, Chandan Gupta and Subhendra Mohanty for discussions. This project has received funding from the European Union's Horizon 2020 research and innovation programme InvisiblesPlus RISE under the Marie SklodowskaCurie grant agreement No 690575. This project has received funding from the European Union's Horizon 2020 research and innovation programme Elusives ITN under the Marie Sklodowska-Curie grant agreement No 674896.

Open Access. This article is distributed under the terms of the Creative Commons Attribution License (CC-BY 4.0), which permits any use, distribution and reproduction in any medium, provided the original author(s) and source are credited.

\section{References}

[1] F. Capozzi, E. Lisi, A. Marrone, D. Montanino and A. Palazzo, Neutrino masses and

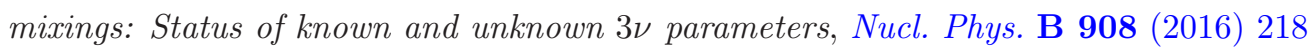
[arXiv: 1601.07777] [INSPIRE].

[2] I. Esteban, M.C. Gonzalez-Garcia, M. Maltoni, I. Martinez-Soler and T. Schwetz, Updated fit to three neutrino mixing: exploring the accelerator-reactor complementarity, JHEP 01 (2017) 087 [arXiv:1611.01514] [INSPIRE].

[3] T2K collaboration, K. Abe et al., Combined Analysis of Neutrino and Antineutrino Oscillations at T2K, Phys. Rev. Lett. 118 (2017) 151801 [arXiv:1701.00432] [INSPIRE].

[4] NOvA collaboration, P. Adamson et al., First measurement of electron neutrino appearance in NOvA, Phys. Rev. Lett. 116 (2016) 151806 [arXiv:1601.05022] [INSPIRE].

[5] NOvA collaboration, P. Adamson et al., Constraints on Oscillation Parameters from $\nu_{e}$ Appearance and $\nu_{\mu}$ Disappearance in NOvA, Phys. Rev. Lett. 118 (2017) 231801 [arXiv: 1703.03328] [INSPIRE].

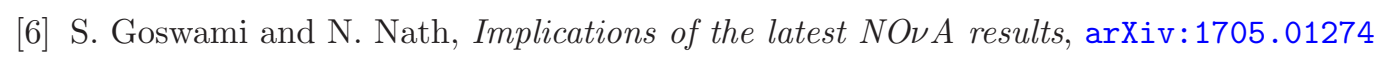
[INSPIRE].

[7] A. Acker, S. Pakvasa and J.T. Pantaleone, Decaying Dirac neutrinos, Phys. Rev. D 45 (1992) 1 [inSPIRE].

[8] A. Acker and S. Pakvasa, Solar neutrino decay, Phys. Lett. B 320 (1994) 320 [hep-ph/9310207] [INSPIRE].

[9] G.B. Gelmini and M. Roncadelli, Left-Handed Neutrino Mass Scale and Spontaneously Broken Lepton Number, Phys. Lett. B 99 (1981) 411 [INSPIRE].

[10] Y. Chikashige, R.N. Mohapatra and R.D. Peccei, Are There Real Goldstone Bosons Associated with Broken Lepton Number?, Phys. Lett. B 98 (1981) 265 [INSPIRE].

[11] S. Pakvasa, Do neutrinos decay?, AIP Conf. Proc. 542 (2000) 99 [hep-ph/0004077] [INSPIRE].

[12] C.W. Kim and W.P. Lam, Some remarks on neutrino decay via a Nambu-Goldstone boson, Mod. Phys. Lett. A 5 (1990) 297 [INSPIRE].

[13] A. Acker, A. Joshipura and S. Pakvasa, A neutrino decay model, solar anti-neutrinos and atmospheric neutrinos, Phys. Lett. B 285 (1992) 371 [INSPIRE]. 
[14] M. Lindner, T. Ohlsson and W. Winter, A combined treatment of neutrino decay and neutrino oscillations, Nucl. Phys. B 607 (2001) 326 [hep-ph/0103170] [INSPIRE].

[15] P. Coloma and O.L.G. Peres, Visible neutrino decay at DUNE, arXiv:1705.03599 [InSPIRE].

[16] A.M. Gago, R.A. Gomes, A.L.G. Gomes, J. Jones-Perez and O.L.G. Peres, Visible neutrino decay in the light of appearance and disappearance long baseline experiments, JHEP 11 (2017) 022 [arXiv: 1705.03074] [INSPIRE].

[17] J.N. Bahcall, N. Cabibbo and A. Yahil, Are neutrinos stable particles?, Phys. Rev. Lett. 28 (1972) 316 [INSPIRE].

[18] Z.G. Berezhiani, G. Fiorentini, M. Moretti and A. Rossi, Fast neutrino decay and solar neutrino detectors, Z. Phys. C 54 (1992) 581 [InSPIRE].

[19] Z.G. Berezhiani, M. Moretti and A. Rossi, Matter induced neutrino decay and solar anti-neutrinos, Z. Phys. C 58 (1993) 423 [INSPIRE].

[20] S. Choubey, S. Goswami and D. Majumdar, Status of the neutrino decay solution to the solar neutrino problem, Phys. Lett. B 484 (2000) 73 [hep-ph/0004193] [INSPIRE].

[21] A. Bandyopadhyay, S. Choubey and S. Goswami, MSW mediated neutrino decay and the solar neutrino problem, Phys. Rev. D 63 (2001) 113019 [hep-ph/0101273] [INSPIRE].

[22] A.S. Joshipura, E. Masso and S. Mohanty, Constraints on decay plus oscillation solutions of the solar neutrino problem, Phys. Rev. D 66 (2002) 113008 [hep-ph/0203181] [INSPIRE].

[23] A. Bandyopadhyay, S. Choubey and S. Goswami, Neutrino decay confronts the SNO data, Phys. Lett. B 555 (2003) 33 [hep-ph/0204173] [INSPIRE].

[24] R. Picoreti, M.M. Guzzo, P.C. de Holanda and O.L.G. Peres, Neutrino Decay and Solar Neutrino Seasonal Effect, Phys. Lett. B 761 (2016) 70 [arXiv:1506.08158] [InSPIRE].

[25] J.M. Berryman, A. de Gouvêa and D. Hernandez, Solar Neutrinos and the Decaying Neutrino Hypothesis, Phys. Rev. D 92 (2015) 073003 [arXiv:1411.0308] [InSPIRE].

[26] J.A. Frieman, H.E. Haber and K. Freese, Neutrino Mixing, Decays and Supernova Sn1987a, Phys. Lett. B 200 (1988) 115 [INSPIRE].

[27] J.M. LoSecco, What the atmospheric neutrino anomaly is not, hep-ph/9809499 [INSPIRE].

[28] V.D. Barger, J.G. Learned, S. Pakvasa and T.J. Weiler, Neutrino decay as an explanation of atmospheric neutrino observations, Phys. Rev. Lett. 82 (1999) 2640 [astro-ph/9810121] [INSPIRE].

[29] P. Lipari and M. Lusignoli, On exotic solutions of the atmospheric neutrino problem, Phys. Rev. D 60 (1999) 013003 [hep-ph/9901350] [INSPIRE].

[30] G.L. Fogli, E. Lisi, A. Marrone and G. Scioscia, Super-Kamiokande data and atmospheric neutrino decay, Phys. Rev. D 59 (1999) 117303 [hep-ph/9902267] [INSPIRE].

[31] S. Choubey and S. Goswami, Is neutrino decay really ruled out as a solution to the atmospheric neutrino problem from Super-Kamiokande data?, Astropart. Phys. 14 (2000) 67 [hep-ph/9904257] [INSPIRE].

[32] V.D. Barger, J.G. Learned, P. Lipari, M. Lusignoli, S. Pakvasa and T.J. Weiler, Neutrino decay and atmospheric neutrinos, Phys. Lett. B 462 (1999) 109 [hep-ph/9907421] [INSPIRE].

[33] Super-Kamiokande collaboration, Y. Ashie et al., Evidence for an oscillatory signature in atmospheric neutrino oscillation, Phys. Rev. Lett. 93 (2004) 101801 [hep-ex/0404034] [INSPIRE]. 
[34] M.C. Gonzalez-Garcia and M. Maltoni, Status of Oscillation plus Decay of Atmospheric and Long-Baseline Neutrinos, Phys. Lett. B 663 (2008) 405 [arXiv:0802.3699] [INSPIRE].

[35] R.A. Gomes, A.L.G. Gomes and O.L.G. Peres, Constraints on neutrino decay lifetime using long-baseline charged and neutral current data, Phys. Lett. B 740 (2015) 345 [arXiv: 1407.5640] [INSPIRE].

[36] S. Choubey, D. Dutta and D. Pramanik, Constraining invisible neutrino decay from T2K and NOvA, work in progress (2018).

[37] T. Abrahão, H. Minakata, H. Nunokawa and A.A. Quiroga, Constraint on Neutrino Decay with Medium-Baseline Reactor Neutrino Oscillation Experiments, JHEP 11 (2015) 001 [arXiv: 1506. 02314] [INSPIRE].

[38] J.F. Beacom, N.F. Bell, D. Hooper, S. Pakvasa and T.J. Weiler, Decay of high-energy astrophysical neutrinos, Phys. Rev. Lett. 90 (2003) 181301 [hep-ph/0211305] [INSPIRE].

[39] M. Maltoni and W. Winter, Testing neutrino oscillations plus decay with neutrino telescopes, JHEP 07 (2008) 064 [arXiv:0803.2050] [INSPIRE].

[40] S. Pakvasa, A. Joshipura and S. Mohanty, Explanation for the low flux of high energy astrophysical muon-neutrinos, Phys. Rev. Lett. 110 (2013) 171802 [arXiv:1209.5630] [INSPIRE].

[41] G. Pagliaroli, A. Palladino, F.L. Villante and F. Vissani, Testing nonradiative neutrino decay scenarios with IceCube data, Phys. Rev. D 92 (2015) 113008 [arXiv:1506.02624] [INSPIRE].

[42] M. Bustamante, J.F. Beacom and K. Murase, Testing decay of astrophysical neutrinos with incomplete information, Phys. Rev. D 95 (2017) 063013 [arXiv: 1610.02096] [INSPIRE].

[43] J.M. Berryman, A. de Gouvêa, D. Hernández and R.L.N. Oliveira, Non-Unitary Neutrino Propagation From Neutrino Decay, Phys. Lett. B 742 (2015) 74 [arXiv:1407.6631] [INSPIRE].

[44] A.M. Dziewonski and D.L. Anderson, Preliminary reference Earth model, Phys. Earth Planet Int. 25 (1981) 297.

[45] DUNE collaboration, R. Acciarri et al., Long-Baseline Neutrino Facility (LBNF) and Deep Underground Neutrino Experiment (DUNE) Conceptual Design Report Volume 1: The LBNF and DUNE Projects, arXiv:1601.05471 [INSPIRE].

[46] DUNE collaboration, R. Acciarri et al., Long-Baseline Neutrino Facility (LBNF) and Deep Underground Neutrino Experiment (DUNE) Conceptual Design Report Volume 2: The Physics Program for DUNE at LBNF, arXiv:1512.06148 [INSPIRE].

[47] DUNE collaboration, J. Strait et al., Long-Baseline Neutrino Facility (LBNF) and Deep Underground Neutrino Experiment (DUNE) Conceptual Design Report Volume 3: Long-Baseline Neutrino Facility for DUNE, arXiv:1601.05823 [INSPIRE].

[48] DUNE collaboration, R. Acciarri et al., Long-Baseline Neutrino Facility (LBNF) and Deep Underground Neutrino Experiment (DUNE) Conceptual Design Report, Volume 4 The DUNE Detectors at LBNF, arXiv:1601.02984 [INSPIRE].

[49] P. Huber, M. Lindner and W. Winter, Simulation of long-baseline neutrino oscillation experiments with GLoBES (General Long Baseline Experiment Simulator), Comput. Phys. Commun. 167 (2005) 195 [hep-ph/0407333] [INSPIRE].

[50] P. Huber, J. Kopp, M. Lindner, M. Rolinec and W. Winter, New features in the simulation of neutrino oscillation experiments with GLoBES 3.0: General Long Baseline Experiment Simulator, Comput. Phys. Commun. 177 (2007) 432 [hep-ph/0701187] [INSPIRE].

[51] S.K. Raut, Effect of non-zero $\theta_{13}$ on the measurement of $\theta_{23}$, Mod. Phys. Lett. A 28 (2013) 1350093 [arXiv:1209.5658] [INSPIRE]. 